
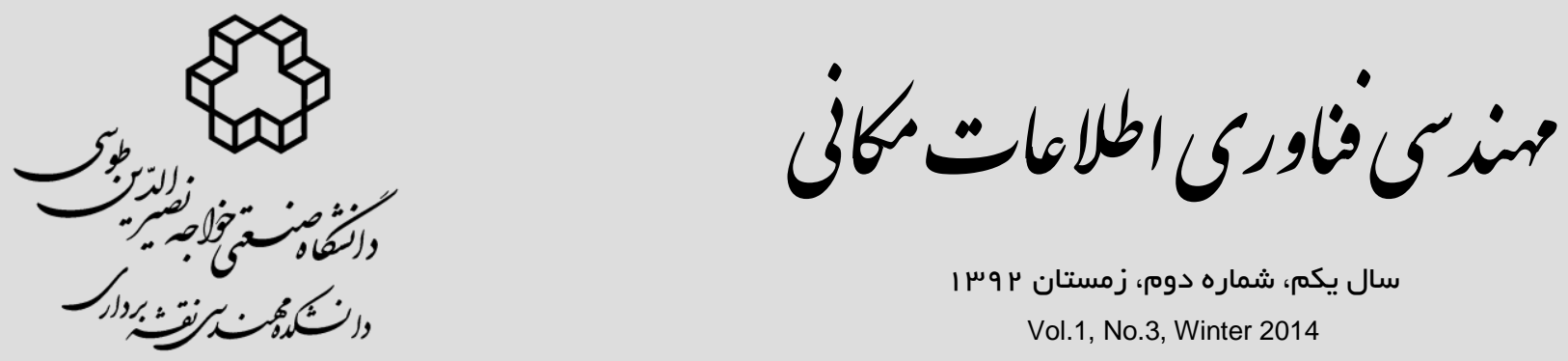

يهنهبندى حساسيت زمين لغزش با استفاده از مدل احتمالاتى وزن واقعه

\author{
حميدرضا يورقاسمى'، حميدرضا مرادى '*، مجيد محمدى'

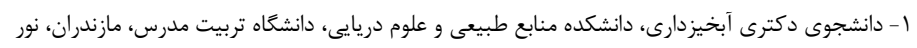

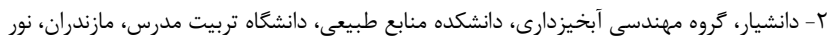

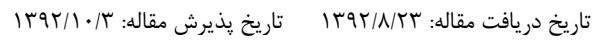

جكيده

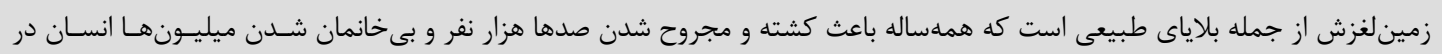

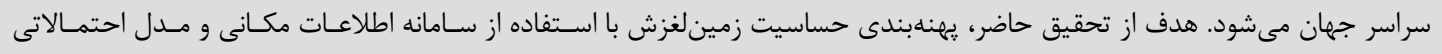
Weight of Evidence

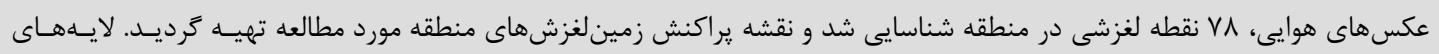

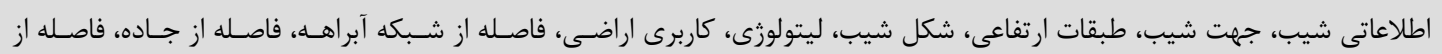

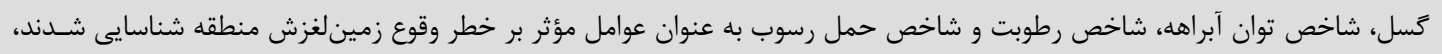

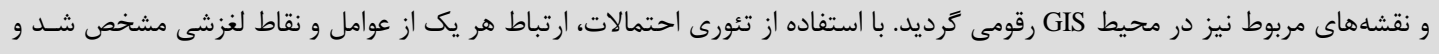

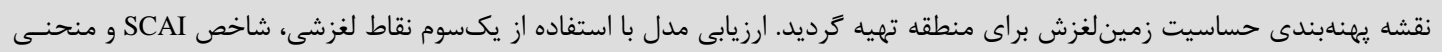

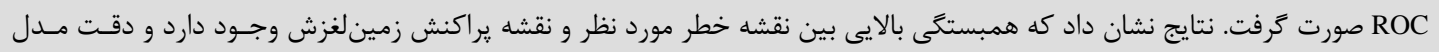

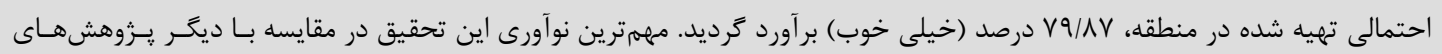

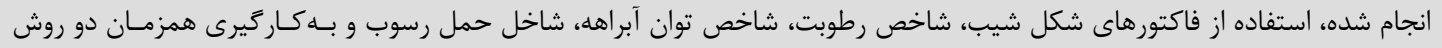

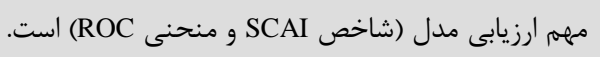

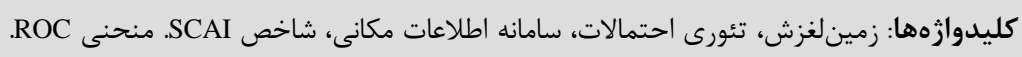




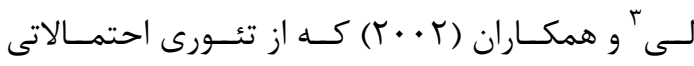
Weight of Evidence استفاده كردند. در اين تحقيق از با عامـل مـؤثر بـــاى

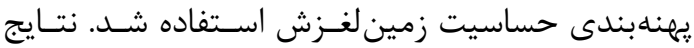
ارزيابى نشان داد كه مدل مذكور دقت بالايى در منطقه

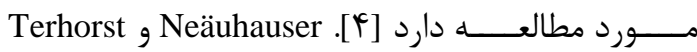
(Y.VV) حساسيت زمــينلغـزش از مـدل Weight of Evidence

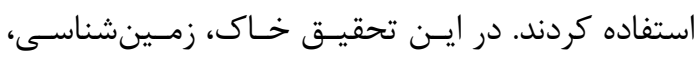

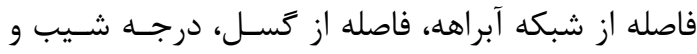

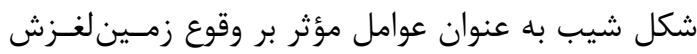

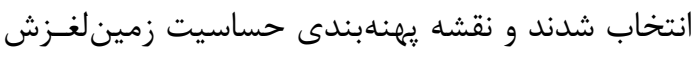

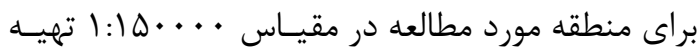

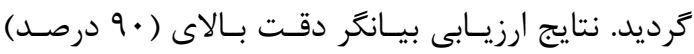

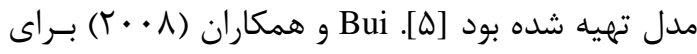

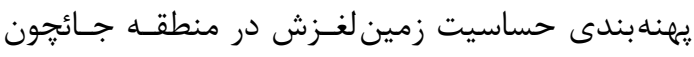

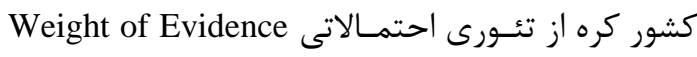

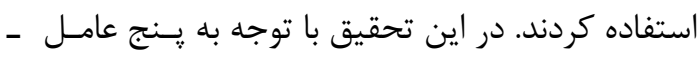
درجه شيب، جهت شيب، ارتفـاع، ليتولـوزى و يوشـش كياهى ـ نقشه خطر زمين لغزش تهيه كرديد [9].

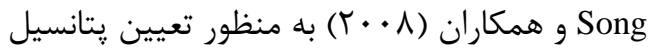

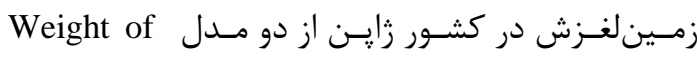
Evidence

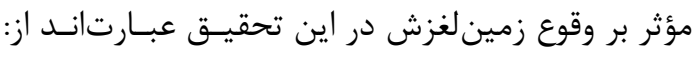

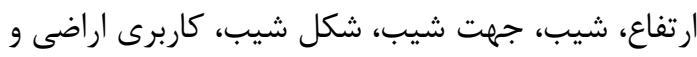
Contributing Area Contributing استفاده از تمامى عوامل، حذف عوامـل Area

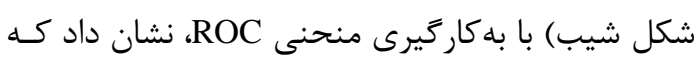
رويكرد دوم و سوم مدل سازى با مدل احتمـالاتى داراى

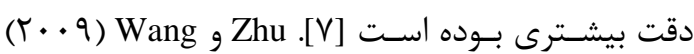

1. Bayesian

2. Expert knowledge

3. Lee

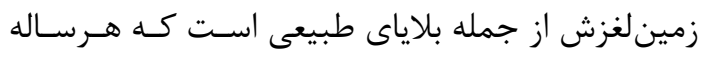
باعث كشته و مجروح شدن صدها هزار نفر و بى خانمان شدن ميليونها فرد در سراسر جهان مىشود. خسارت ندار

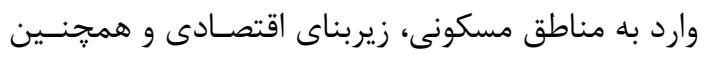

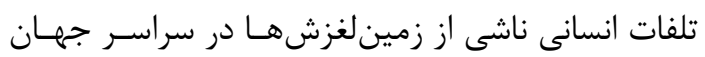

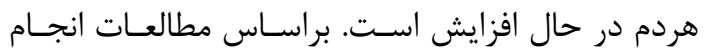

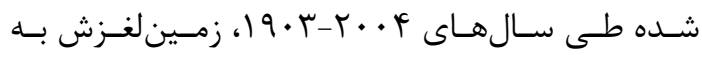

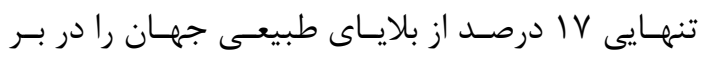

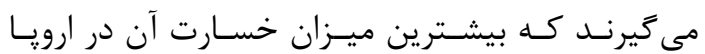

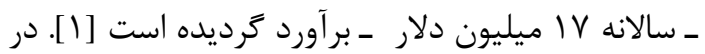

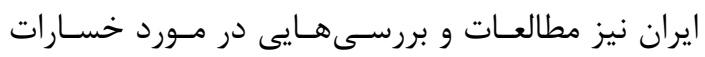

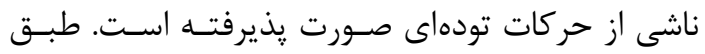

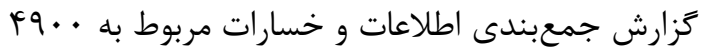

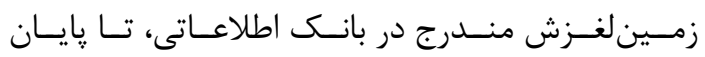

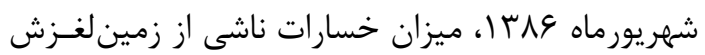

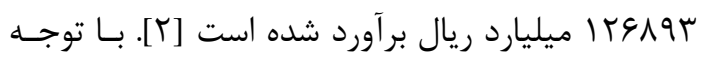

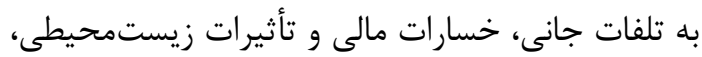

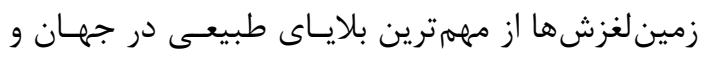
بلهويزه در كشور ما به شمار مى آيند كه هر ساله تأثيرات عمدهاى در تخريب جادههاى ارتباطى، تخريسب مراتع،

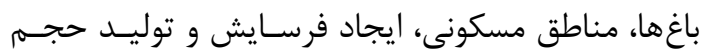

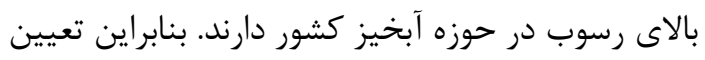

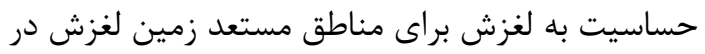

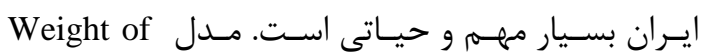

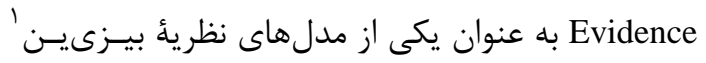

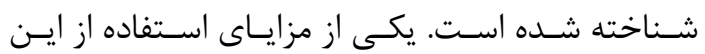

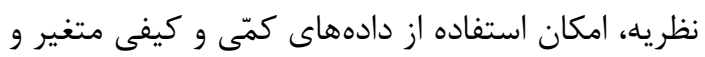

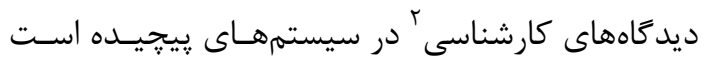

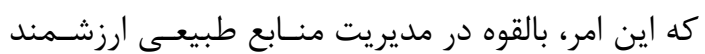

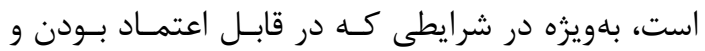

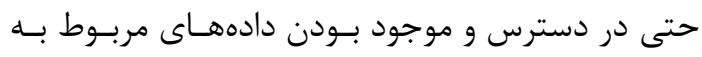

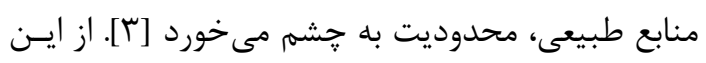
رو در يهنهبندى خطر زمينلغزش نيز مطالعات مختلفى محدي

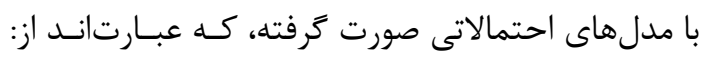


زمرؤ مهمترين و ير آبتــرين رودهــاى اسـتان مازنــدران

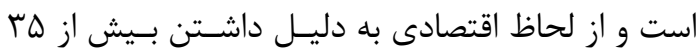
كاركاه يرورش ماهى و آبيارى مـزارع در سـطح جلخـهـ،

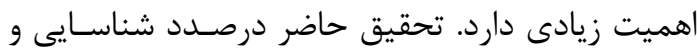
تعيين مناطق حساس به زمين لغزش با استفاده از روش

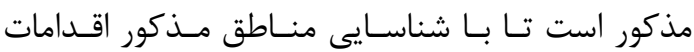
اجرايى براى كنترل در منطقه، سمت و سـويى منطقى

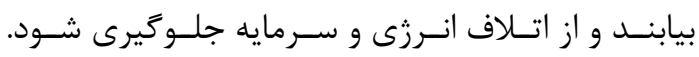

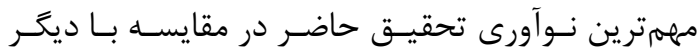

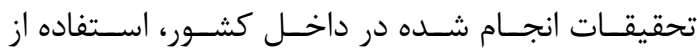

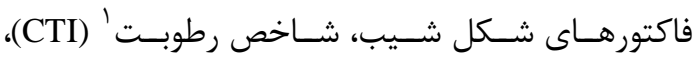

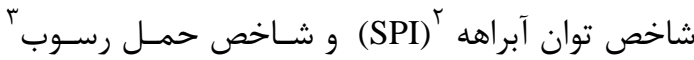

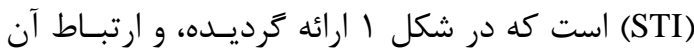
با زمينلغزشهاى منطقه مورد مطالعه نيز در جـدول 1 الندان

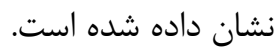

بهمنظور تهيه نقشه حساسيت زمين لغـزش از دو مـدل

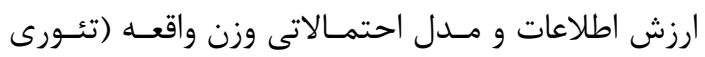
بيزىين) استفاده كردند. نقشه حساسيت زمين لغزش با

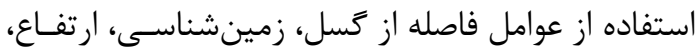

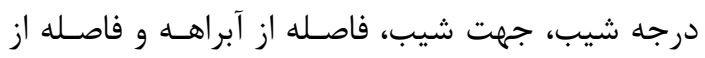
جاده تهيه گرديد. نتايج ارزيابى نشان داد كه به ترتيسب

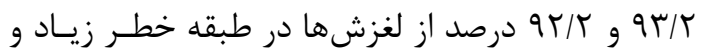

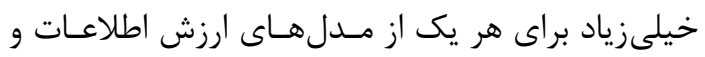

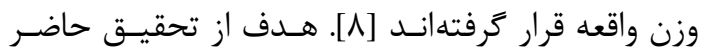

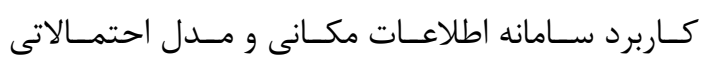
Weight of Evidence

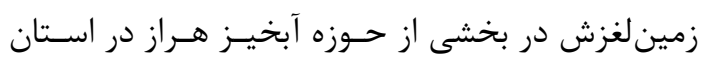

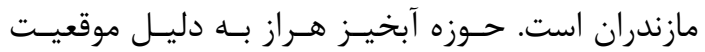

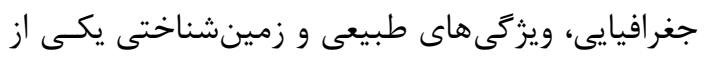

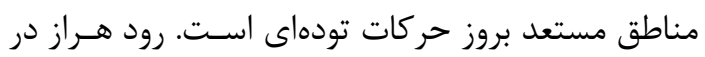

جدول ا. ارتباط بين هر يك از عوامل مؤثر و نقاط لغزشى با استفاده از مدل احتمالى Weight of Evidence

\begin{tabular}{|c|c|c|c|c|c|c|c|c|c|c|c|c|}
\hline $\mathbf{C} / \mathbf{S}(\mathbf{C})$ & $\mathbf{S}(\mathbf{C})$ & $\mathbf{S}^{2}\left(\mathbf{W}^{*}\right)$ & $\mathbf{S}^{2}\left(\mathbf{W}^{+}\right)$ & $\mathrm{C}$ & $\mathbf{W}^{-}$ & $\mathbf{W}^{+}$ & لغزش & لعزشاد & درصد بيكسلهاي لغزشي & تعاد بيكسل لغزشاي & كلاس & $\frac{y}{z}$ \\
\hline.$/ \varepsilon 1$ & $1 / \cdot 1$ &.$/ \cdot r$ & 1 &.$/ \Sigma 1$ &.$\%$ &.$/ \Sigma 1$ & I/Ar & 1 & $1 / K 1$ & $1 \% \wedge 01$ & .0 & \multirow{6}{*}{$\frac{\text { 争: }}{3}$} \\
\hline.$- / 7 \pi$ &.$/ N T$ &.$/ \cdot r$ & .10 & $-\cdot / \leqslant 0$ &.$/ \cdot r$ & $-\cdot / \leqslant r$ & $\Gamma / \tau \varepsilon$ & r & $0 / 7 r$ & ТะYTA & 7.10 & \\
\hline .199 &.$/ 1 \% 0$ &.$/ \cdot r$ &.$/ 1$ &.$/ \Gamma \xi$ & -.1 .0 &.$/ 199$ & $1 \wedge / 1 \wedge$ & 1. & $1 \% / 09$ & $1007 . Y$ & $17-4$. & \\
\hline.$/ V T$ &.$/ \gamma^{\prime}$ &.$/ \cdot r$ & .1 .0 &.$/ Y 1$ &.$/ \cdot v$ &.$/ 1 \varepsilon$ & $r \leqslant / 00$ & 19 & $r . / \cdot r$ & 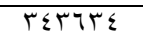 & $r \cdot .0$. & \\
\hline.$- /$ AT &.$/ 40$ &.$/ \cdot r$ & .11 &.$- / \times 9$ & .1 .7 &.$- / Y T$ & $1 / / 1 \wedge$ & 1. & $r Y / 9)$ & TAYIV & $0 .-V$. & \\
\hline-.10 &.$/ T Y$ &.$/ \cdot r$ &.$/ \cdot 1$ &.$- / 17$ &.$/ \cdot \xi$ & $-\cdot / / Y$ & $T \Gamma / T \varepsilon$ & $\pi$ & $r T / T \varepsilon$ & $r \cdot \leqslant \wedge . q$ & $v \cdot<$ & \\
\hline$-\cdot / \wedge \Lambda$ & $\cdot / \Sigma V$ &.$/ \cdot r$ &.$/ 5$ & $--\cdot|\varepsilon|$ & .1 .0 & $-. \cdot / \Gamma V$ & $9 / .9$ & 0 & $M T / T$ & $1 \leqslant 999 V$ & شمال & \multirow{8}{*}{ 䒠: } \\
\hline$-. / 1 \leqslant$ & ד4 & $\cdot / \cdot Y$ &.$/ 11$ & -.1 .0 & .1 .1 & $-\cdot / \cdot \varepsilon$ & $17 / \% 4$ & 9 & $\mathrm{IV} / \cdot \mathrm{V}$ & 1904.1 & شمال شرقي & \\
\hline$-1 / 79$ & $\cdot / V Y$ &.$/ \cdot \mathrm{r}$ & .10 & $-I / Y Y$ &.$/ \cdot 1$ & $-1 / 14$ & $r / \tau \varepsilon$ & $r$ & $11 / \times 9$ & $14917 \%$ & شرق & \\
\hline r/AO &.$/ \mu$. &.$/ \cdot r$ & .1 .7 & .110 & $-\cdot / 1 \wedge$ &.$/ \mathrm{TV}$ & r9/.9 & 17 & $1 \leqslant / 90$ & $|V| \mid \leq \varepsilon$ & جنوب شرقي & \\
\hline$-1 / \varepsilon r$ & .109 & $\cdot / \cdot Y$ & "זr/ & $-\because / 10$ &.$/ \cdot \mathrm{V}$ & $-\cdot / V \lambda$ & $0 / 57$ & $\Gamma$ & $11 / 10$ & 1TOTVY & جنوب & \\
\hline $1 / T r$ & ד4 &.$/ \cdot Y$ &.$/ 11$ & $\cdot \mid / \Sigma)$ &.- .1 .7 &.$/ 1 \% 0$ & $17 / 4 \pi$ & 9 & $11 / 01$ & $1 \pi 1 \times 11$ & جنوب غربي & \\
\hline $1 / 7 \varepsilon$ &.$/ \Sigma$. &.$/ \cdot \mathrm{r}$ &.$/ 1 \varepsilon$ &.$/ 77$ &.$- / .7$ &.$/ 7$. & IT/NT & V & $7 / 99$ & V99V9 & غرب & \\
\hline$-1 / \% \wedge$ & $.10 \mathrm{Y}$ & $\cdot / \cdot r$ &.$/ T_{0}$ &.$- / 77$ &.$/ \cdot \mathrm{V}$ &.$- / 7$. & $V / Y V$ & $\varepsilon$ & $T / K r$ & $101 Y 91$ & شمال غربي & \\
\hline
\end{tabular}

جدول ا. ارتباط بين هر يك از عوامل مؤثر و نقاط لغزشى با استفاده از مدل احتمالى Weight of Evidence (ادامه)

\begin{tabular}{|c|c|c|c|c|c|c|c|c|c|c|c|c|}
\hline $\mathbf{C} / \mathbf{S}(\mathbf{C})$ & S(C) & $\mathbf{S}^{2}\left(\mathbf{W}^{-}\right)$ & $S^{2}\left(\mathbf{W}^{+}\right)$ & C & $\mathbf{W}^{-}$ & $\mathbf{W}^{+}$ & لدزش & لغزش & درصد بيّكسلهاي & تعاداد بيكسل لغزاي & كلاس & $\frac{y}{g}$ \\
\hline
\end{tabular}

1. (CTI) Compound Topographic Index or Topographic Witness Index

2. (SPI) Stream Power Index

3. (STI) Sediment Transport Index 
نشريه علمى يثوهشى - مهندسى فناورى اطلاعات مكانى

سال اول • شماره دوم • زمستان م وس

\begin{tabular}{|c|c|c|c|c|c|c|c|c|c|c|c|c|}
\hline$\infty$ & $\infty$ &.$/ . \mathrm{r}$ & $\infty$ & . &.$/ .4$ & $\infty$ & . & . & $r / \varepsilon q$ & $r \wedge \leq 7 r$ & $1, \ldots-10 \ldots$ & \multirow{7}{*}{$\begin{array}{l}\frac{y}{3} \\
\overline{3} \\
\frac{7}{4}\end{array}$} \\
\hline$\varepsilon / K r$ & $\cdot / \mathrm{r}$ &.$/ \cdot r$ & .1 .0 & $1 / T$. & $-\cdot / r \wedge$ &.$/ 94$ & $\Gamma \leqslant / 0 \varepsilon$ & 19 & $I T / V T$ & $10 \% \cdot 11$ & $10 \ldots-11 \ldots$ & \\
\hline$r / Y \varepsilon$ &.$/ \mathrm{rA}$ & .1 .4 & .1 .0 &.$/ 74$ & $-\cdot / T$. & $\cdot / \varepsilon 1$ & $\varepsilon$. & TY & $r \tau / \varepsilon \lambda$ & $r . r .0 \Lambda$ & $|\wedge \ldots-r| \ldots$ & \\
\hline$-Y / Y V$ &.$/ \varepsilon$. &.$/ \cdot Y$ & $\cdot /$. &.$- / 94$ &.$/ \mathrm{V}$ &.$- \cdot V \varepsilon$ & $I Y / V Y$ & $v$ & $r T / V T$ & $r .0 \Lambda \leqslant \leqslant$ & $r l \cdots-r \leqslant \ldots$ & \\
\hline$-1 / \mu_{\Lambda}$ & $\cdot / \varepsilon r$ &.$/ \cdot \mathrm{r}$ & $\cdot / \mathrm{VV}$ &.$- / 7$. & .1 .9 &.- .101 & $1 . / 91$ & 7 & $1 \Lambda / r$. & T.ATrI & $r \varepsilon \cdots-r V \cdots$ & \\
\hline$-1 / \wedge \Lambda$ & $1 / \cdot 1$ &.$/ \cdot Y$ & 1 & $-1 / 19$ &.$/ 1$ & $-1 / \lambda$. & I/AY & 1 & 1.197 & TrOHAE & $r V \cdots$ & \\
\hline$\infty$ & $\infty$ &.$/ \cdot Y$ & $\infty$ & - &.$/ \cdot 1$ & $\infty$ & . & . & $1 / \leqslant Y$ & 17194 & $\mu \ldots<$ & \\
\hline$r / I \varepsilon$ &.$/ T V$ & $\cdot / \cdot \varepsilon$ & $.1 \cdot 4$ & .101 & $-\cdot / T V$ &.$/ \mu 1$ & $0 \leqslant 100$ & $r$. & $\varepsilon \cdot / 19$ & Fo9q1F & A & \multirow{5}{*}{ 註 } \\
\hline$-1 / 7 V$ & .109 &.$/ \cdot Y$ & 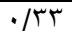 &.$- / 99$ & .1 .9 &.$- / 9$. & $0 / \leqslant 0$ & $r$ & $T \pi / \varepsilon \pi$ & 104741 & B & \\
\hline$-1 / 9$. & $\cdot / V Y$ &.$/ \cdot Y$ & .10 & $-1 / T V$ &.$/ 1$ & $-1 / \% 7$ & $\Gamma / \tau \varepsilon$ & $r$ & $1 Y / \wedge \Lambda$ & $1 \leqslant V \Gamma \wedge T$ & $\mathrm{C}$ & \\
\hline$\infty$ & $\infty$ &.$/ \cdot \mathrm{r}$ & $\infty$ & . &.$/ \cdot r$ & $\infty$ & . & . & I/NY & 19700 & $\mathrm{D}$ & \\
\hline$\cdot / \mathrm{VT}$ & $\cdot / \mathrm{r}$ & .1 .4 & .1 .0 & $\cdot / r$. & $-. \cdot / \cdot V$ &.$/ 1 T$ & ד ד/אד & r. & TI/VA & rqTV.0 & $\mathrm{E}$ & \\
\hline .1 .0 & . &.$/ \cdot Y$ & $\cdot / \cdot 1$ &.$/ \cdot Y$ &.$- / \cdots r$ &.$/ .1$ & $\overline{Y I / A Y}$ & Tr & TI/00 & $r \leqslant 77.1$ & مرتع خوب & \multirow{4}{*}{$\begin{array}{c}y \\
7 \\
y \\
\bar{y} \\
\bar{y}\end{array}$} \\
\hline$\varepsilon / 7 \curlywedge$ & $\cdot / \gamma \Lambda$ &.$/ . r$ & .1 .0 & $1 / \pi$ & ו & $1 /$. & ד"ז/ז & r. & سא/זו & $10 Y 011$ & كثاورزي باغو و & \\
\hline $1 / \lambda$. & $1 / \cdot 1$ &.$/ \cdot \mathrm{r}$ & 1 & $1 / \lambda 1$ &.$- / \cdot r$ & $1 / \lambda$. & I/AY & 1 &.$/ \Gamma$. & $r \leqslant 0$. & مسكونى & \\
\hline$-r / 79$ &.$/ K \Lambda$ &.$/ .4$ & .1 .0 & $-1 / \cdot Y$ & .104 &.$- / \leqslant \wedge$ & $\varepsilon$. & TY & $T \varepsilon / A Y$ & $V \&|V| Y$ & مرتع متوسط & \\
\hline .101 & .109 &.$/ \cdot Y$ & 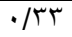 & $\cdot / \Gamma \xi$ &.$- / \cdot r$ & 每 & $0 / \leqslant 0$ & $r$ & $r / 94$ & $\varepsilon \leqslant q \leqslant r$ & $--1 \ldots$ & \multirow{5}{*}{$\frac{.9}{\frac{\pi}{3}} \frac{7}{3}$} \\
\hline $1 / \Gamma \varepsilon$ & $.10 Y$ &.$/ \cdot Y$ &.$/$ Yo &.$/ 79$ & $-. \cdot / \cdot \varepsilon$ &.$/ 77$ & $V / K V$ & $\varepsilon$ & $r / V V$ & $\varepsilon M \mid r r$ & I. & \\
\hline$r / \tau r$ & $\cdot / \varepsilon r$ &.$/ \cdot Y$ &.$/ \mathrm{VV}$ & $1 / 1 \varepsilon$ & $-. \cdot / \cdot 1$ & $1 / .7$ & $1 . / 91$ & 7 & $r / V V$ & $\varepsilon \pi \mid \leqslant \varepsilon$ & $r \ldots r \cdots$ & \\
\hline.$- / 11$ & $\cdot / V Y$ &.$/ \cdot r$ & .10 & $-. \cdot / \cdot 1$ & $\cdot \% r$ & $-. \cdot / \cdot 1$ & $\Gamma / \tau \varepsilon$ & $r$ & $T / 7 r$ & $\varepsilon \leqslant 91 \leqslant$ & $\Gamma \cdots-\ldots$ & \\
\hline$-r / r q$ &.$/ \Gamma$ &.$/ \cdot \mathrm{V}$ &.$/ \cdot r$ & $-\cdot / V Y$ & $.10 \mathrm{~V}$ & -.110 & VY/VT & $\varepsilon$. & $\Lambda \varepsilon / 71$ & $97 \lambda 1 \leqslant 9$ & $\varepsilon \cdots<$ & \\
\hline O/АT & $\cdot / \mathrm{r} \wedge$ & .1 .0 & .1 .4 & $1 / 71$ &.$- / 70$ &.$/ 97$ & 7. & Tा & $r r / \cdot r$ & TATONE & $\cdot-1 \ldots$ & \multirow{5}{*}{$\frac{\sqrt{9}}{\frac{7}{7}} \frac{7}{\frac{3}{3}}$} \\
\hline$-1 / 7 V$ & $\cdot / \Sigma V$ &.$/ \cdot Y$ &.$/ T$ &.$- / \mathrm{VA}$ & $\cdot / 1$. &.$- / 7 \wedge$ & $9 / .9$ & 0 & $18 / 91$ & T.0V09 & I..r. & \\
\hline -. &.$/ \varepsilon$. &.$/ \cdot Y$ & $\cdot /$./ & $-. \cdot / 11$ &.$/ \cdot 1$ &..$- / .9$ & $T Y / V r$ & $\mathrm{v}$ & $1 \Gamma / 9 V$ & 1091.1 & r...r. & \\
\hline$-I / T V$ & .109 &.$/ \cdot Y$ &.$/ 4 \pi$ & $-\cdot / \wedge \mid$ &.$/ \cdot \mathrm{V}$ & $-\cdot / V \leqslant$ & $0 / \leqslant 0$ & $r$ & $11 / \leqslant 9$ & $1 \pi \mid \leqslant r$. & $\Gamma \cdots-\varepsilon$ & \\
\hline$-r / \cdot V$ & $\cdot / \varepsilon$. &.$/ \cdot Y$ & $\cdot / 1 \varepsilon$ & $-1 / Y \varepsilon$ &.$/ T V$ &.$- / 9 V$ & $I Y / N T$ & V & rr/or & TATVIV & $\varepsilon \cdots<$ & \\
\hline $7 / 11$ &.$/ T V$ &.$/ \cdot \mathrm{r}$ &.$/ \cdot \varepsilon$ & $1 / 7 V$ & $-\cdot / \xi 1$ & $1 / K 4$ & $\Sigma / / A r$ & TT & $11 / 9$. & ITTYA & $\cdot-1 \ldots$ & \multirow{6}{*}{$\frac{.9}{\frac{\pi}{7}} \frac{1}{1}$} \\
\hline-.109 & $.10 Y$ & .1 .4 &.$/ T_{0}$ & $-\cdot / / T$ & .1 .5 &.$- / \Gamma \wedge$ & $V / K V$ & $\varepsilon$ & $9 / 7 \varepsilon$ & $11 . \mathrm{rAr}$ & $\ldots r \ldots$ & \\
\hline.$/ Y_{0}$ & $\cdot / \varepsilon V$ &.$/ \cdot Y$ & $\cdot / K$ &.$/ 1 Y$ &..$- / .1$ &.$/ 11$ & $9 / 1$. & 0 & N/IV & $q r \leqslant \leqslant$. & r...r. & \\
\hline.- .104 & .109 &.$/ \cdot r$ & . & $-\cdot \cdot / r T$ &.$/ \cdot r$ & $-\cdot / \mu$. & $0 / \leqslant 0$ & $r$ & $V / \mu r$ & ArAVฯ & $r \cdots-\ldots$ & \\
\hline -. & .109 &.$/ \cdot \mathrm{r}$ &.$/ 4 \pi$ & $-.0 / 19$ &.$/ \cdot 1$ &.$- / 1 \wedge$ & $0 / \leqslant 0$ & $r$ & $T / 0 Y$ & $V \leqslant T Y T$ & $\varepsilon \ldots-0 \ldots$ & \\
\hline$-r / 7 \leqslant$ &.$/ 199$ &.$/ \cdot r$ & $.1 \cdot 7$ & $-1 / .7$ & $\cdot / \leqslant 7$ &.$- / 7$ & $r \cdot / 91$ & IV & $07 / \Sigma \varepsilon$ & $T \leqslant O \wedge Y \wedge$ & $0 \ldots<$ & \\
\hline $9 / \cdot r$ &.$/ \Sigma V$ &.$/ r$ &.$/ \cdot r$ & $\varepsilon / Y \varepsilon$ & $-Y / Y T$ & $1 / 9 V$ & $9 . / 91$ & 0. & $1 Y / 7 \pi$ & $1 \leqslant \leqslant 0 Y_{q}$ & $\cdot-\varepsilon$ & \multirow{4}{*}{$\begin{array}{c}3 \\
3 \\
-17 \\
-7 \\
.7\end{array}$} \\
\hline$-\Lambda / \Gamma^{\prime}$ & $.10 Y$ &.$/ \cdot Y$ &.$/$ ro & - & $1 / 19$ & $-r / \leqslant Y$ & $V / K V$ & $\varepsilon$ & $10 / 97$ & 9АrनY। & $\varepsilon_{-1}$ & \\
\hline$\cdot / K T$ & $1 / .1$ &.$/ \cdot Y$ & 1 & 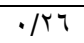 & $-\cdot / \cdots \varepsilon$ &.$/ Y 7$ & I/AY & 1 & $1 / \varepsilon$. & $17.8 V$ & N-IT & \\
\hline$\infty$ & $\infty$ &.$/ . \mathrm{r}$ & $\infty$ & . & $.1 \ldots 0$ & $\infty$ & - & . & $\%$ & $0 \leqslant$ & $M<$ & \\
\hline.$/ 79$ &.$/ \pi$. & .1 .4 &.$/ \cdot \mathrm{V}$ & $\cdot / K 1$ &.$- / .0$ &.$/ 17$ & $T V / T V$ & 10 & $r T / r T$ & Y7797Y & . - r. & \multirow{6}{*}{$\begin{array}{l}3 \\
\frac{3}{3} \\
3 \\
\frac{3}{3} \\
\frac{3}{3} \\
\frac{3}{3}\end{array}$} \\
\hline$-. \cdot / \wedge \wedge$ & . &.$/ \cdot r$ &.$/ \cdot 1$ &..$- / .9$ &.$/ \cdot r$ & $-. \cdot / \cdot V$ & $r / / A r$ & IT & $r T / \varepsilon r$ & YTVqYT & $r \cdot-\varepsilon$. & \\
\hline$-\cdot / \varepsilon r$ & $\cdot / \Gamma \wedge$ &.$/ \cdot Y$ &.$/ 14$ & $-\cdot / T V$ &.$/ \cdot r$ & $-\cdot / 1 \leqslant$ & $1 \leqslant / 00$ & $\Lambda$ & $1 T / V Y$ & 191 TYO & $\varepsilon \cdot-7$. & \\
\hline.$- / T Y$ & $\cdot / \varepsilon r$ &.$/ \cdot r$ &.$/ \mathrm{V}$ & -.1 .0 & $.1 \cdots 7$ & -.1 .0 & $1 . / 91$ & 7 & $11 / \varepsilon r$ & $1 r \cdot 71$. & ᄀ.-A. & \\
\hline..$- / 11$ & $.10 Y$ &.$/ \cdot \mathrm{r}$ &.$/ 1 \% 0$ &..$- / .7$ & $\cdot / \cdots \varepsilon$ &.- .1 .0 & $V / K V$ & $\varepsilon$ & $V / T V$ & 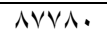 & $\lambda \cdot-1 \ldots$ & \\
\hline$\cdot /$. &.$/ 1 \% 0$ &.$/ . \mathrm{Y}$ &.$/ 1$ & .1 .0 &.$- / 1 \cdot 9$ &.$/ \cdot \varepsilon$ & $1 \wedge / 11$ & 1. & $\mid V / \varepsilon \varepsilon$ & 1997.1 & $1 \cdots<$ & \\
\hline
\end{tabular}

جدول ا. ارتباط بين هر يك از عوامل مؤثر و نقاط لغزشى با استفاده از مدل احتمالى Weight of Evidence (ادامه)

\begin{tabular}{|c|c|c|c|c|c|c|c|c|c|c|c|c|}
\hline $\mathrm{C} / \mathrm{S}(\mathrm{C})$ & $\mathbf{S}(\mathbf{C})$ & $S^{2}\left(W^{*}\right)$ & $\mathbf{S}^{2}\left(\mathbf{W}^{+}\right)$ & C & $\mathbf{W}$ & $\mathbf{W}^{+}$ & لغزش & لغزش & درصد بيكسلهاي لغزشي & تعاقا بيدكسل هلغاي & كلاس & $\frac{y}{g}$ \\
\hline.$/ 94$ &.$/ \pi$. &.$/ . r$ & .1 .7 &.$/ K V$ & $-\cdot / \cdot V$ & $\cdot / r$. & $r 9 / .9$ & 17 & Tr/VV & KVI974 & -1 & \multirow{3}{*}{$\frac{43}{13}$} \\
\hline - & $\cdot / / 99$ &.$/ . r$ & .1 .7 &.$- / . r$ & .1 .1 &.$- / . r$ & $r \cdot / 91$ & IV & TI/74 & TTroo & $1 \cdot-r$. & \\
\hline.$- / T V$ & 每 &.$/ \cdot Y$ & $\cdot / \cdot 1$ &..$- / .9$ & $\cdot / \cdot Y$ & $-\cdot \cdot / \cdot V$ & $r / / A Y$ & IT & $r r / r q$ & YTV7I9 & r.-r. & \\
\hline$-r / v$. & $\cdot / \varepsilon V$ &.$/ \cdot \mathrm{r}$ & $\cdot / r$ & 更 &.$/ . r$ & $-\cdot / 1 \times 9$ & $9 / .9$ & 0 & $T K / T$. & $1 T 90 \wedge r$ & $\Gamma \cdot-\varepsilon$. & 3 \\
\hline$\cdot / V Y$ & $.10 Y$ &.$/ \cdot \mathrm{r}$ &.$/$ KO &.$/ T V$ &.$- / \cdot r$ &.$/ \% 0$ & $V / T V$ & $\varepsilon$ & $0 / 1 \pi$ & ONVTY & $\varepsilon \cdot-0$. & 3 \\
\hline.$- / V V$ & $1 / .1$ &.$/ . \mathrm{r}$ & 1 &.$- / \mathrm{VV}$ &.$/ . r$ & $-. / 1 \times 0$ & I/AY & 1 & $r / 10$ & $\varepsilon \varepsilon \mid r V$ & $0 .<$ & \\
\hline
\end{tabular}




\begin{tabular}{|c|c|c|c|c|c|c|c|c|c|c|c|c|}
\hline$-1 / 0$ & $\cdot / \Gamma \Lambda$ &.$/ . r$ & .1 .0 & $-\cdot / \leqslant Y$ & $\cdot / 1 \Lambda$ & $\mid-\cdot / r \varepsilon$ & $r N / / \Lambda$ & r) & $\varepsilon N / \Gamma_{0}$ & OORTYV & مقعر & 3 \\
\hline $1 / 0$. & . / r & .1 .0 &.$/ .4$ & •/\& & $-\cdot / r \varepsilon$ &.$/ 1$ & $T I / A Y$ & ro & $01 / 70$ & $091.0 \leqslant$ & محدب & 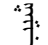 \\
\hline
\end{tabular}
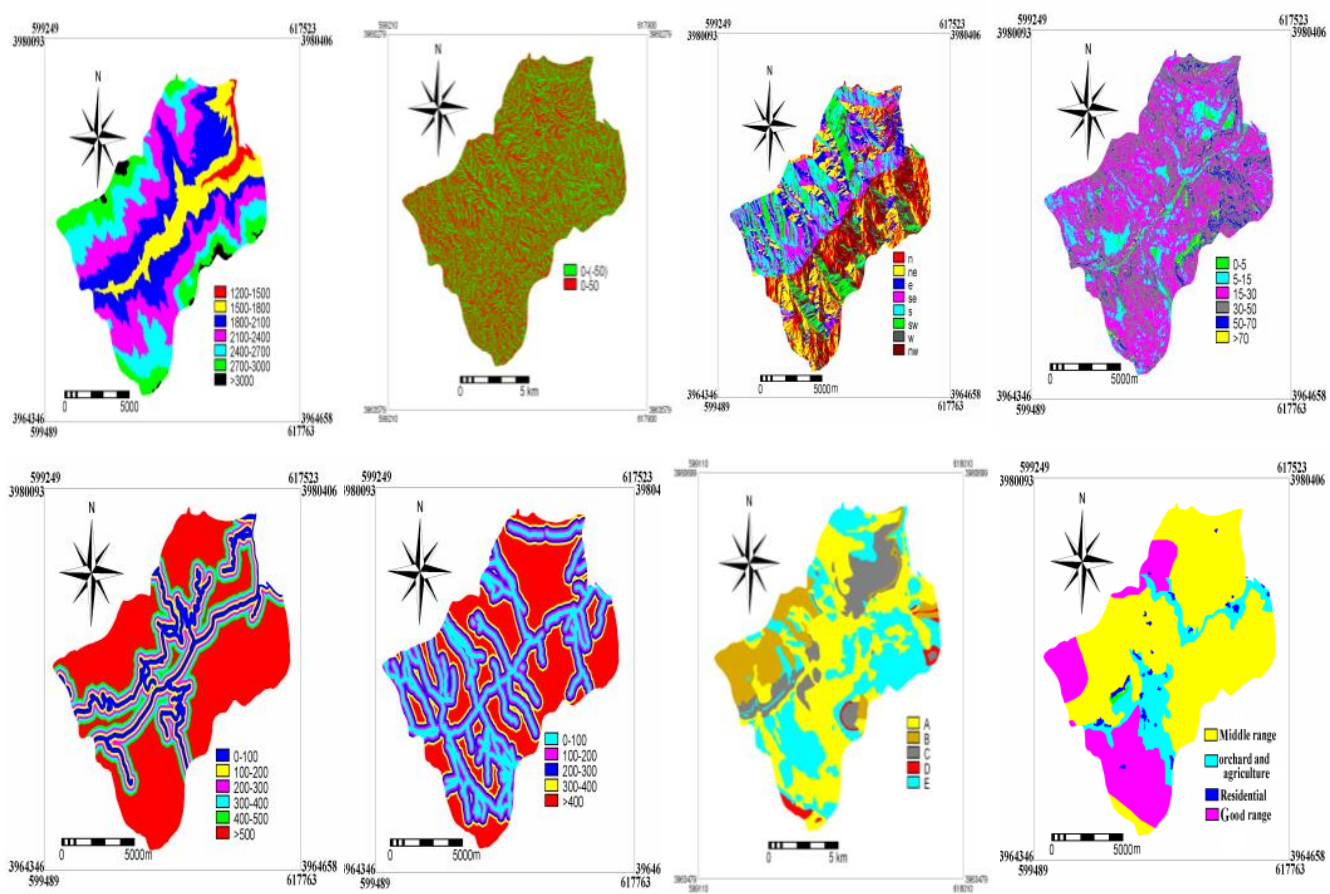

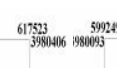
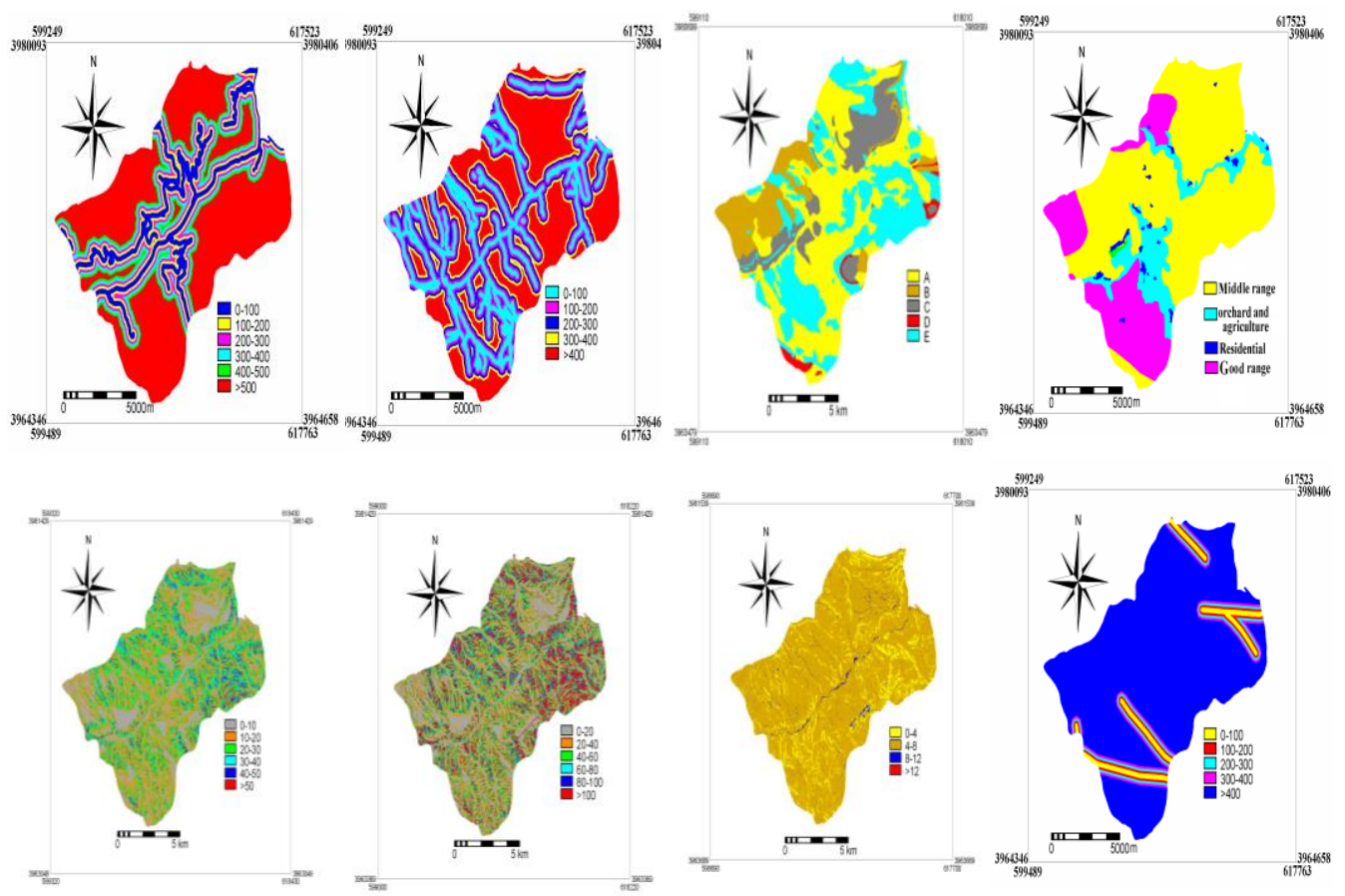

شكل ا. به ترتيب از راست به جِ نقشه شيب، جهت شيب، شكل شيب، ارتفاع، كاربرى اراضى، ليتولوزى، فاصله از آبراهه،

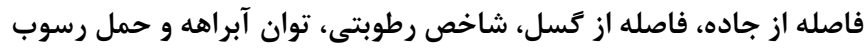

(كمّى و كيفى) به طور همزمان استفاده شده است.

$$
\text { r- r- بواد و روشها }
$$

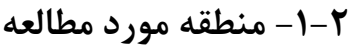

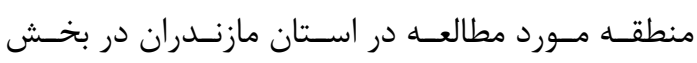

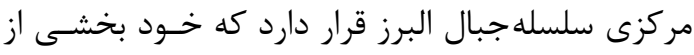

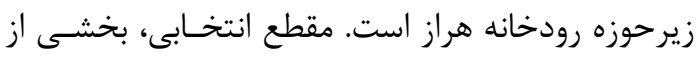

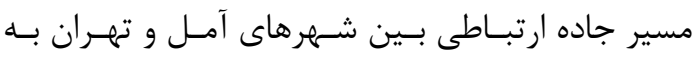

عوامل ياد شده، به همراه ديخر عوامل در يهنهابندى

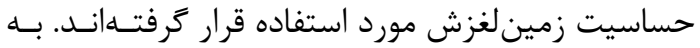

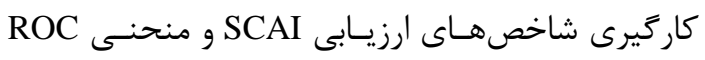

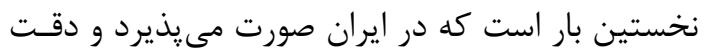

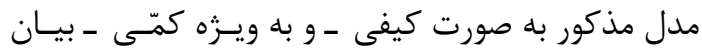

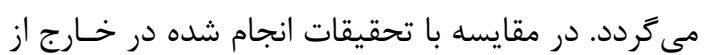

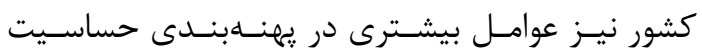

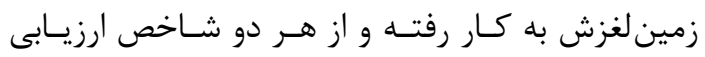


مربوط به آن به ترتيب از راست به جٍِ (نقشـه شـيب،

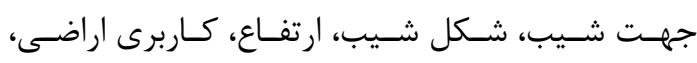

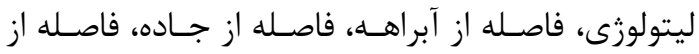
كسل، شاخص رطوبت، شاخص توان آبراهـه و شـاخص آجساده

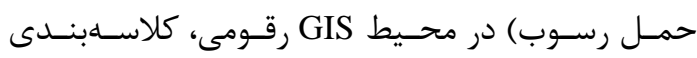

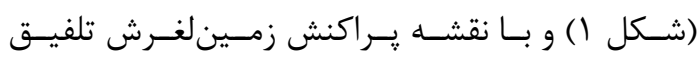

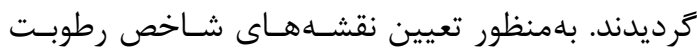

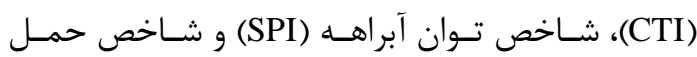

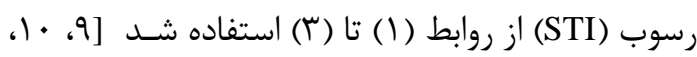

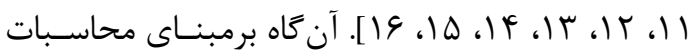

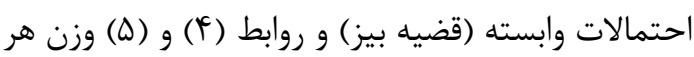
عامل مربوط به آن تعيين گرديد.

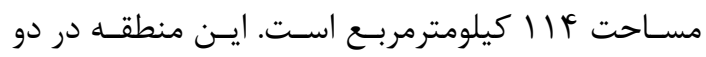

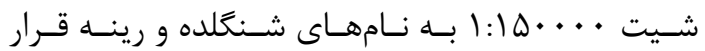

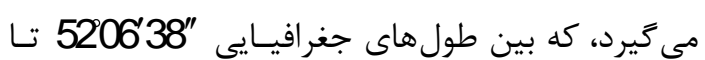
"5217'24" . 3557'11"

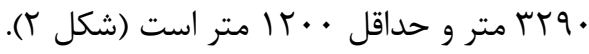

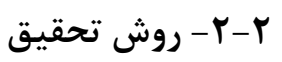

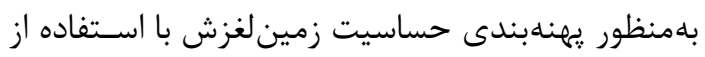

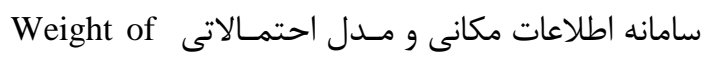

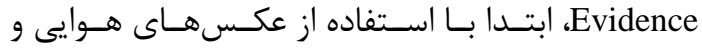

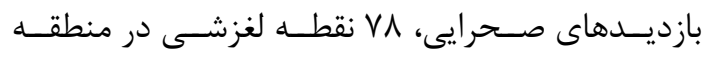

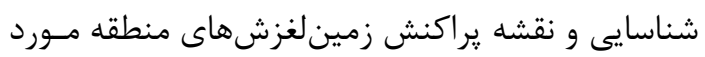

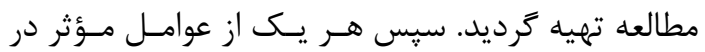

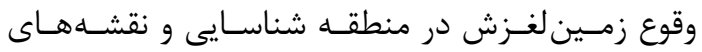

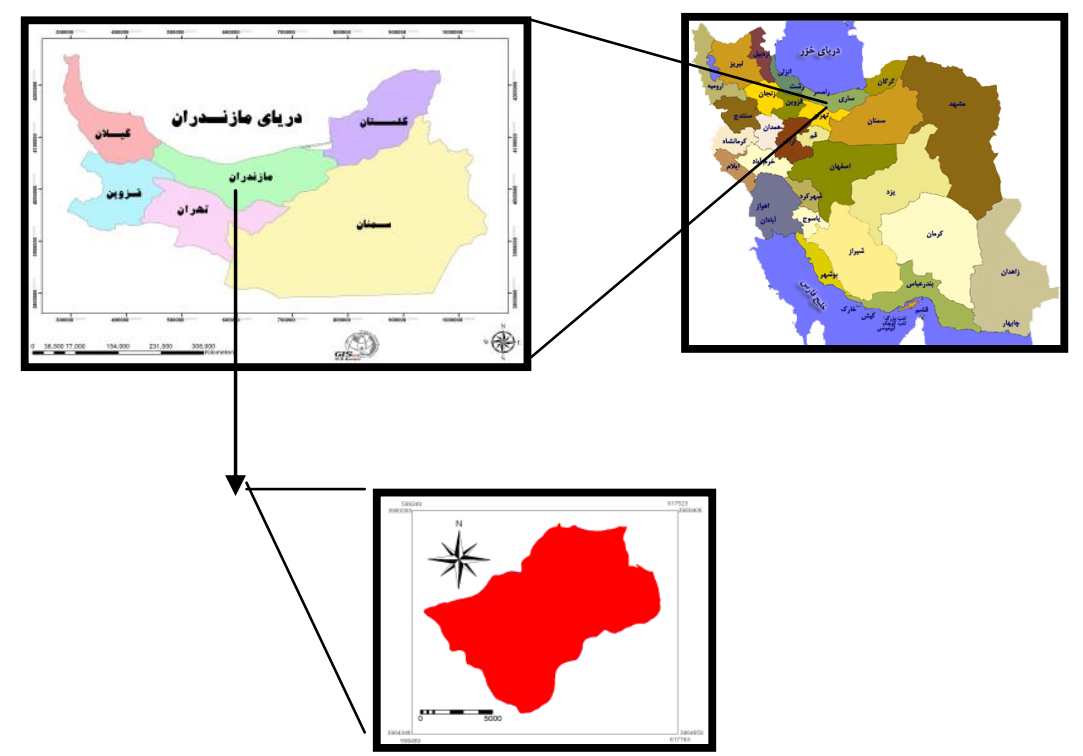

شكل r. موقعيت منطقه مورد مطالعه

شيب برحسب درجه و STI توان حمل آبراهـه و يـا بــهـ

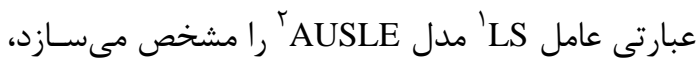

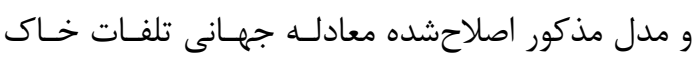
(بSEL)

$\mathrm{w}_{\mathrm{i}}^{+}=\log _{\mathrm{e}}\left[\mathrm{P}\left(\mathrm{B}_{\mathrm{i}} \mid \mathrm{S}\right) / \mathrm{P}\left(\mathrm{B}_{\mathrm{i}} \mid \overline{\mathrm{S}}\right)\right]$

(f) (f) (1ابطه
$\mathrm{CTI}=\ln \left(\frac{\mathrm{A}_{\mathrm{S}}}{\tan \beta}\right)$

(1) (1ابطه (1)

$\mathrm{SPI}=\mathrm{A}_{\mathrm{S}} \cdot \tan \beta$

رابطه (r)

$\mathrm{STI}=\left(\frac{\mathrm{A}_{\mathrm{S}}}{22 / 13}\right)^{0 / 6} \times\left(\frac{\sin \beta}{0 / 0896}\right)^{1 / 3}$

رابطه (r) 


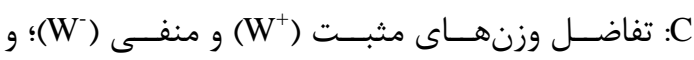

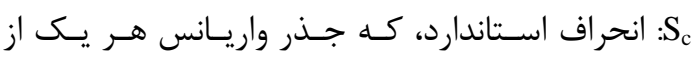
وزنهاى مثبت و منفى است.

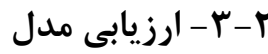

در روش Weight of Evidence بـراى وزندهــى بــــ

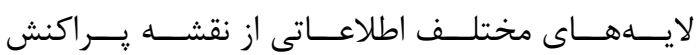

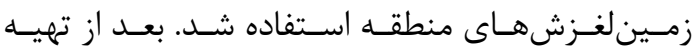

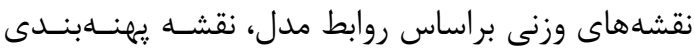

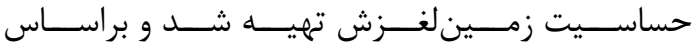

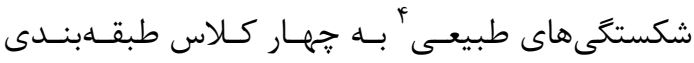

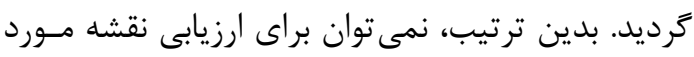

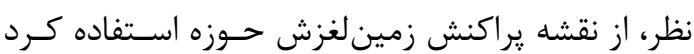

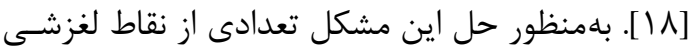

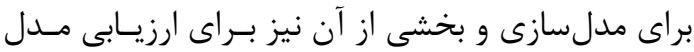

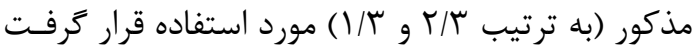

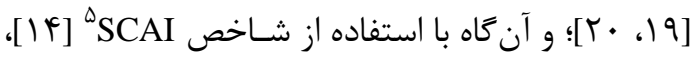

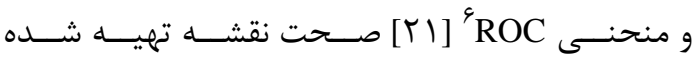
تأييد گرديد.

r- بنتايج

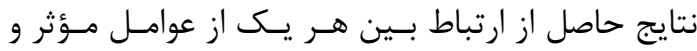

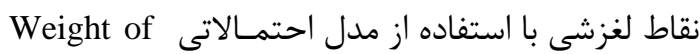

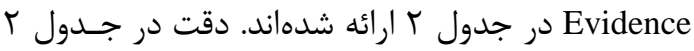

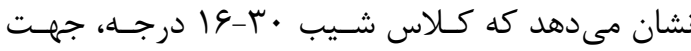

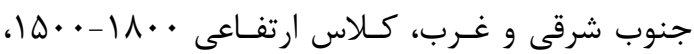

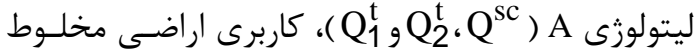

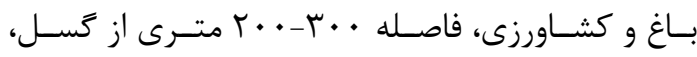

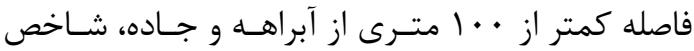

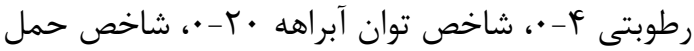

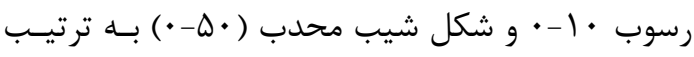

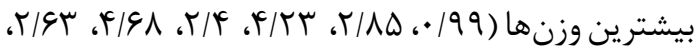

4. Natural Breaks

5. Seed Cell Area Index

6. Relative Operating Characteristics Curve

$$
\mathrm{w}_{\mathrm{i}}^{-}=\log _{\mathrm{e}}\left[\mathrm{P}\left(\bar{B}_{\mathrm{i}} \mid \mathrm{S}\right) / \mathrm{P}\left(\overline{\mathrm{B}}_{\mathrm{i}} \mid \overline{\mathrm{S}}\right)\right] \quad \text { (ه) (هابطه }
$$

به منظور درك هر جه بهتر روابـط و سـهولت كـار، بلهويزه در محـيط GIS، مـدل براسـاس جــدول بـ و بــهـ

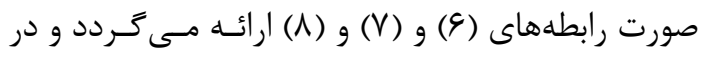

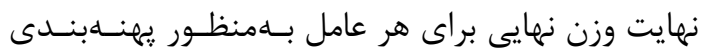
خطر زمين لغزش مورد استفاده قرار گرفت [IV]

جدول r. جههار حالت بتانسيل ايجاد زمين لغزش [r]

\begin{tabular}{|c|c|c|}
\hline & \multicolumn{2}{|c|}{ عوامل ايجاد زمين لغزش } \\
\hline زمين لغزش & حضور & عدم حضور \\
\hline حضور & $\mathrm{N}_{\text {pix } 1}$ & $\mathrm{~N}_{\text {pix2 }}$ \\
\hline عدم حضور & $\mathrm{N}_{\text {pix3 }}$ & $\mathrm{N}_{\text {pix } 4}$ \\
\hline
\end{tabular}

(9)

$\mathrm{W}_{\mathrm{i}}^{+}=\log _{\mathrm{e}}\left[\left(\mathrm{N}_{\text {pix } 1} /\left(\mathrm{N}_{\text {pix } 1}+\mathrm{N}_{\text {pix } 2}\right)\right) /\left(\mathrm{N}_{\text {pix } 3} /\left(\mathrm{N}_{\text {pix } 3}+\mathrm{N}_{\text {pix } 4}\right)\right)\right]$

$$
\text { (V) رابطه }
$$

$\mathrm{W}_{\mathrm{i}}^{-}=\log _{\mathrm{e}}\left[\left(\mathrm{N}_{\text {pix } 2} /\left(\mathrm{N}_{\text {pix } 1}+\mathrm{N}_{\text {pix } 2}\right)\right) /\left(\mathrm{N}_{\text {pix4 }} /\left(\mathrm{N}_{\text {pix } 3}+\mathrm{N}_{\text {pix4 }}\right)\right)\right]$

$$
\text { كـه د د ر آن }
$$

: تعداد ييكسل هاى لغزشى در يك كلاس،

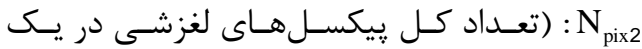

نقشه) - (تعداد ييكسل هاى لغزشى در يك كلاس)،

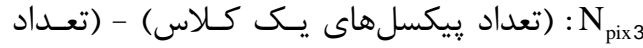
ييكسلهاى لغزشى در يك كلاس)، N Nix4 كل بيكسل هاى لغزشى يك نقشه)- (تعداد ييكسل هاى

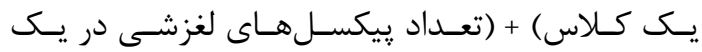
كلاس).
$\mathrm{W}_{\text {final }}=\mathrm{C} / \mathrm{S}_{\mathrm{C}}$
رابطه (1)

كــه در آن، Wfinal : وزن نهــايى اســتاندارد شــده؛

1. Length and Slope

2. Adapted Universal Soil Loss Equation

3. Universal Soil Loss Equation 
ارائه مى كند و بيانگر صحت يا سـقم نقشـهـ يهنــهبنـدى

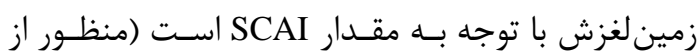
صحت يعنى كاهش مقدار SCAI از كلاس خطر كم بــه سمت كلاس خطر زياد است). دقت در جدول r نشـان معن

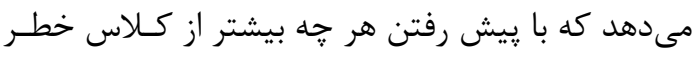

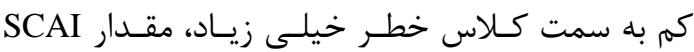

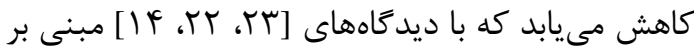
كاهش مقدار SCAI و بهويزه در كلاسهاى خطر بهر زياد و

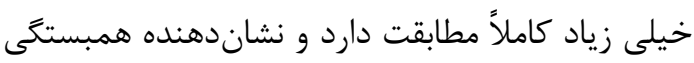
بالاى نقشه حساسيت تهيه شده با نقاط لغزشى موجـون و بازديدهاى صحرايى منطقه مورد مطالعه است.

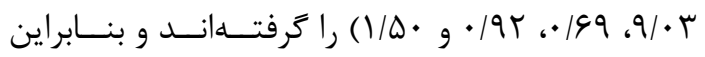
بيشترين حساسيت را در برابر لغـزش در منطقـه مــورد مطالعه دارند. نتايج يهنهبندى حساسيت زمين لغزش بـا استفاده از مدل Weight of Evidence (شكل بل ب) نشـان

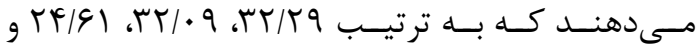
|/11/ درصد از منطقه مورد مطالعـه در كـلاس خطـر كم، متوسط، زياد و خيلى زياد قرار گرفتهاند.

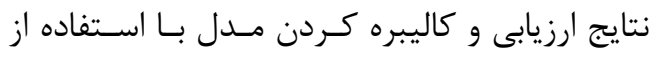
شـاخص SCAI در جــدول r نشـان داده شـده اسـت. شاخص SCAI در حقيقت نسبت درصـد مسـاحت هـر يك از كلاسهاى يهنهبندى حساسيت زمينلغـزش بـهـ درصد لغزش هاى اتفاق افتاده (سب نقطه لغزشى) در هر كلاس است. روش مذكور دقت مدل را به صورت كيفى

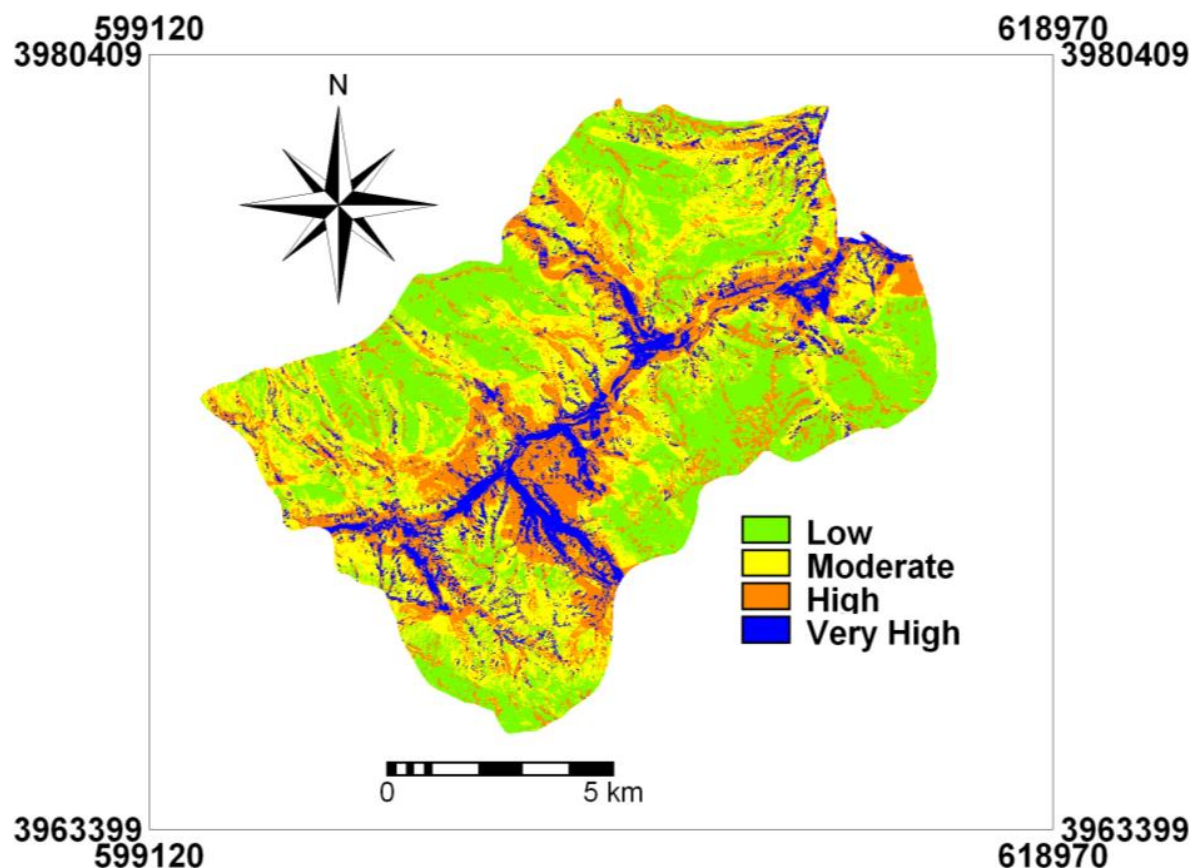

شكل r. نقشه يهنهبندى حساسيت زمين لغزش با استفاده از مدل Weight of Evidence

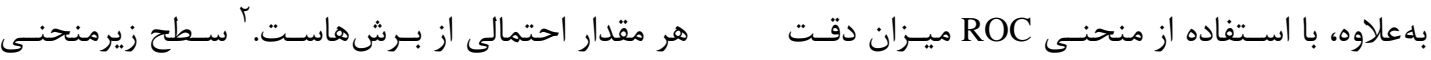
(AUC) ROC

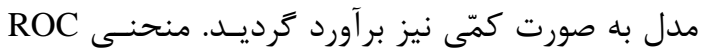
طريـق توصـيف توانـايى آن در تخمـين درسـت وقـايع

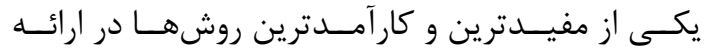

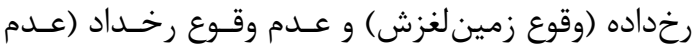
وقوع زمين لغزش) آن است. ايدهآل ترين مدل، بيشترين

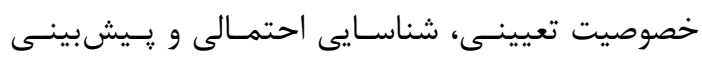

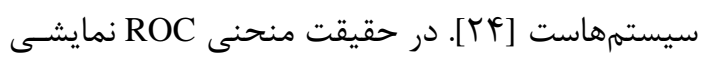
سطح زيرمنحنى را دارد و مقادير AUC از هان • •-1 است. كرافيكى از موازنه' بين نرخ خطاى منفى و مثبت بـراى 


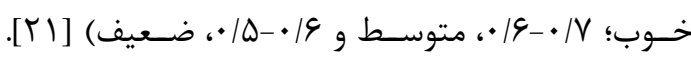
براساس نتايج منحنى ROC، مقـدار سـطح زيرمنحنـى

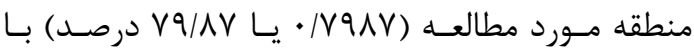

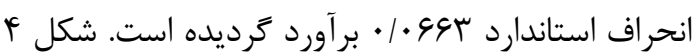
نشاندهنده ارزيابى خيلى خوب مدل احتمالى

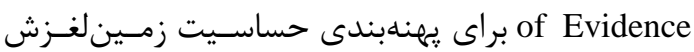

جنانجه مدلى نتواند رخـداد لغزشـى را بهتــر از ديـدگاه

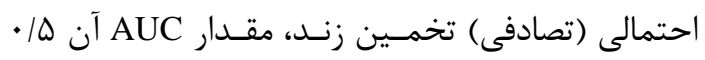
است و زمانى كه منحنى ROC، سطح زير منحنى برابـر

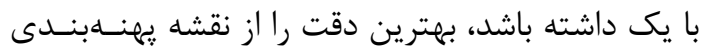
تهيه شده نشان مىدهد. همبستخى كيفى ـ كمّى سطح

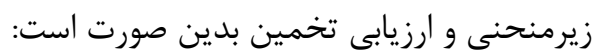

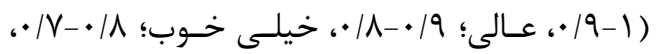

جدول س. نتايج ارزيابى نقشه حساسيت زمين لغزش منطقه مورد مطالعه با استفاده از شاخص SCAI

\begin{tabular}{|c|c|c|c|}
\hline SCAI & درصد Seed & درصد Area & كلاس خطر \\
\hline$r / V I$ & $\Lambda / V$ & $r r / r q$ & كم \\
\hline $1 / \wedge \Delta$ & $1 V / r q$ & $r r / \cdot q$ & متوسط \\
\hline$\cdot|\Lambda|$ & $r \cdot / k r$ & $T F / 9 \mid$ & زياد \\
\hline.$/ T \Delta$ & $F r / F \wedge$ & $11 / \cdot 1$ & خيلى زياد \\
\hline
\end{tabular}

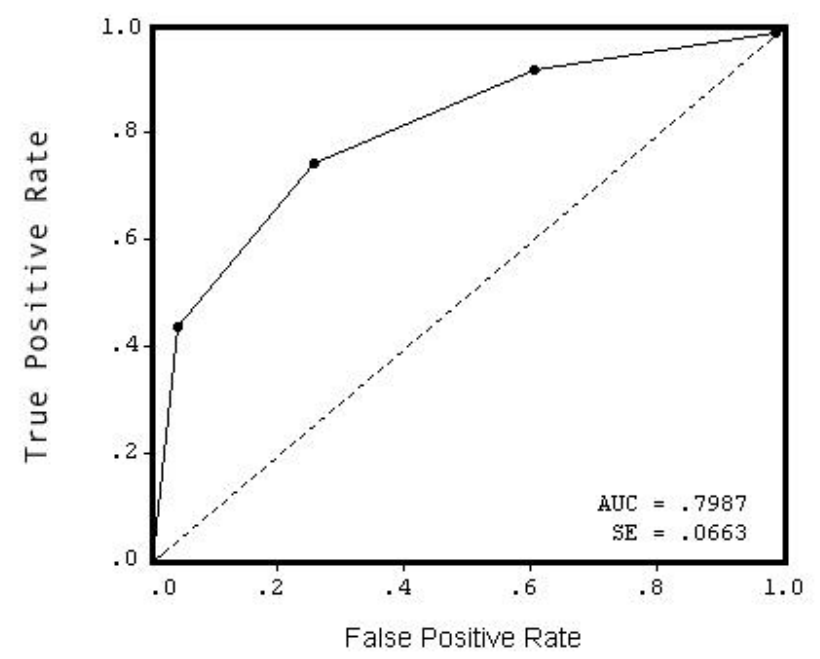

شكل f. منحنى ROC و سطح زير منحنى نقشه يجهنهبندى حساسيت زمين لغزش 
[4] Lee, S., Choi, J. and Min, K., Retrieved from www.adpc.net/casita/case-studieo 2002. Landslide Susceptibility Analysis and Verification Using the Bayesian Probability Model. Environmental Geology, 43, 120-131.

[5] Neuhäuser, B. and Terhorst, B., 2007. Landslide Susceptibility Assessment using "Weights-of-evidence" Applied to a Study Area at the Jurassic Escarpment (SWGermany). Geomorphology, 86, 12-24.

[6] Bui, H.B., Nguyen, Q. and Nguyen, V.T., 2008. GIS-based Weight of Evidence Modeling for Landslide Susceptibility Mapping at Jaechon Area, Korea. In: International Symposium on Geoinformatics for Spatial Infrastructure Development in Earth and Allied Sciences.

[7] Song, R.H., Hiromu, D., Kazutoki, A., Usio, K. and Sumio, M., 2008. Modeling the Potential Distribution of Shallow-seated Landslides Using the Weights of Evidence Method and a Logistic Regression Model: A Case Study of the Sabae Area, Japan. International Journal of Sediment Research, 23, 106-118.

[8] Zhu, C. and Wang, X., 2009. Landslide Susceptibility Mapping: A Comparison of Information and Weights-of Evidence Methods in Three Gorges Area. International Conference on Environmental Science and Information Application Technology, IEEE, DOI 10.1109/ESIAT.2009.187, 342-346.

[9] Moore, I.D., Gessler, P.E., Neslesn, G.A. and Peterson, G.A., 1993. Soil Attribute Prediction using Terrain Analysis. Soil Science Society of American Journal, 57(2), 443-452.

[10] Yesilnacar, E. and Hunter, G.J., 2004. Application of Neural Networks for Landslide Susceptibility Mapping in Turkey. In: J.P. van Leeuwen and H.J.P. Timmermans, eds., Recent Advances in Design and Decision Support Systems in Architecture and Urban Planning, 3-18.

\begin{abstract}
شريه علمى يزوهشى - مهندسى فناورى اطلاعات مكانى

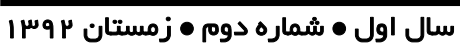

F

تحقيق حاضر با هدف يهرنهبندى حساسيت زمين لغـزش

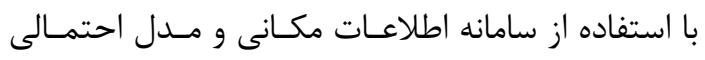
Weight of Evidence

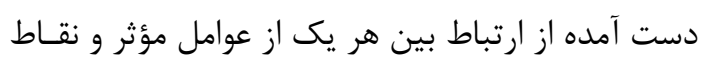

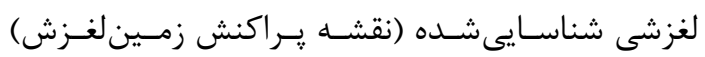

مى توان جنين جمعبندى كرد كه كلاس شـيب • r-19

درجه، جهـت جنـوب شـرقى و غـرب، كـلاس ارتفـاعى ، رهـ

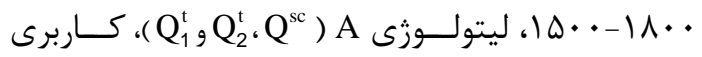

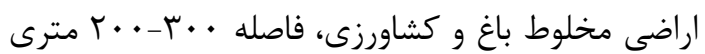

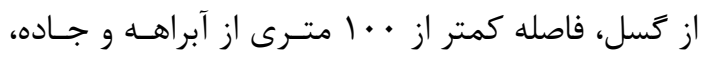

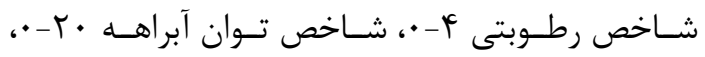

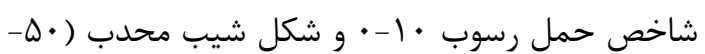

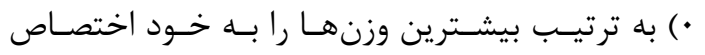

مى دهند و بنابراين بيشترين حساسيت را بـهـ لغـزش در

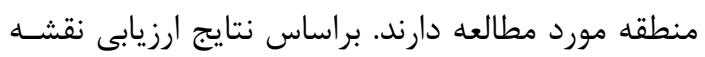

يههنه بندى حساسيت زمين لغزش مى توان بيان كرد كـه

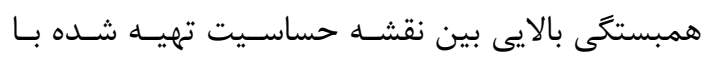

نقاط لغزشى موجود و بازديدهاى صحرايى منطقه مــورد

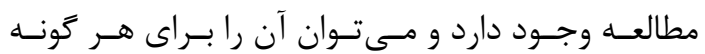

اقدامات مديريتى در منطقه مورد مطالعه در نظر گرفت.

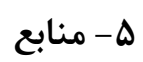

[1] Koehorst, B.A.N., Kjekstad, O., Patel, D., Lubkowski, Z., Knoeff, J.G. and Akkerman, G.J., 2005. Work Package 6, Determination of Socio-Economic Impact of Natural Disasters. Assessing socio-economic Impact in Europe, p.173.

[2] Landslide Working Party, 2007. Iranian Landslides List, Forest. Rangeland and Watershed Association, p.60.

[3] Shahabi, M. and Sadoddin, A., 2009. Bayesian Decision Network Approach for Predicting Impacts of Drought Management Actions in Dry Land Wheat Areas of Golestan Province. In: 5Th National Conference of watershed management, Iran, 3140-3149.
\end{abstract}




\section{-}

[11] Gokceoglu, C., Sonmez, H., Nefeslioglu, H.A., Duman, T.Y. and Can, T., 2005. The March 17, 2005 Kuzulu Landslide (Sivas, Turkey) and Landslide Susceptibility Map of Its Near Vicinity. Eng Geo, 181(1):65-83.

[12] Nefeslioglu, H.A., Gokceoglu, C. and Sonmez, H., 2008. An Assessment on the Use of Logistic Regression and Artificial Neural Networks with Different Sampling Strategies for the Preparation of Landslide Susceptibility Maps. Engineering Geology, 97, 171-191.

[13] Yilmaz, I., 2009. Landslide Susceptibility Mapping using Frequency Ratio, Logistic Regression, Artificial Neural Networks and Their Comparison: A Case Study from Kat Landslides (Tokat-Turkey). Comp Geosc, 35 (6), 1125-1138

[14] Akgun, A. and Needet, T., 2010. Landslide Susceptibility Mapping for Ayvalik (Western Turkey) and its Vicinty by Multi Criteria Decision Analysis. Environ Earth Science, 61, 595-611.

[15] Poudyal, C.P., Chang, C., Oh, H.J. and Lee, S., 2010. Landslide Susceptibility Maps Comparing Frequency Ratio and Artificial Neural Networks: A case Study from the Nepal Himalaya. Environ Earth Sci, 61, 1049-1064.

[16] Regmi, N.R., Giardino, J.R. and Vitek, J.D., 2010. Modeling Susceptibility to Landslides using the Weight of Evidence Approach: Western Colorado, USA. Geomorphology, $115,172-187$.

[17] Van Western, C.J., 2002. Use of Weights of Evidence Modeling for Landslide Susceptibility Mapping, [lecture notes], Retriered from www.adpc.net/casita/casestudies p. 21.
[18]Remendo, J., Gonzales, A., Teran, J., Cendrero, A., Fabbri, A. and Chung, C., 2003. Validation of Landslide Susceptibility Maps, Examples and Applications from a Case Study in Northern Spain, Natural Hazard, 437-449.

[19] Pourghasemi, H.R, Moradi, H.R., Mohammadi, M. and Mahdavifar, M.R., 2009. Landslide Hazard Susceptibility Mapping and its Evaluation Using the Fuzzy Operators. Journal Science and Technology Agriculture and Natural Resources, 12(46A), 375-390.

[20] Pourghasemi, H.R., Moradi, H.R. Fatemi Aghda, S.M. Mahdavifar, M.R. and Mohammdi, M., 2009. Landslide Hazard Assessment Using Fuzzy Multi Criteria Decision- Making Method. Iran-Watershed Management Science and Engineering, 3(8), 51-63.

[21] Yesilnacar, E.K., 2005. The Application of Computational Intelligence to Landslide Susceptibility Mapping in Turkey. Ph.D. Thesis, Department of Geomatics the University of Melbourne, p. 423.

[22] Suzen, M.L. and Doyuran, V., 2004. A Comparision of the GIS based Landslide Susceptibility Assessment Method: Multivariate Versus Bivariate. Environmental Geology, 71, 303-321.

[23] Kincal, C., Akgun, A. and Koca, M.Y., 2009. Landslide Susceptibility Assessment in the Izmir (West Anatolia, Turkey) City Center and Its Near Vicinity by the Logistic Regression Method. Environmental Earth Science, Doi: 10.1007/s12665-009-0070-0.

[24] Swets, J.A., 1988. Measuring the Accuracy of Diagnostic Systems. Science, 240, 1285-1293. 


\title{
Landslide Susceptibility Zoning, Using Weight of Evidence Probabilistic Model
}

\author{
Pourghasemi H.R. ${ }^{1}$, Moradi H.R. ${ }^{2}$, Mohammadi M. ${ }^{1}$ \\ 1- Ph.D. Candidate, Watershed Management Engineering, College of Natural Resources and Marine Science, Tarbiat Modares University, Mazandaran \\ 2- Associate Prof., Dep. of Watershed Management Engineering, College of Natural Resources and Marine Sciences, Tarbiat Modares University
}

\begin{abstract}
Landslide is among the natural disasters which cause many damages such as killing and wounding many peoples and lead to homelessness millions of households every year all over the world. The main objective of this research is making landslides susceptibility zoning, using Geospatial Information System (GIS) and probability model "weight of evidence" in limited areas of Haraz watershed located in Mazandaran province. Due to the mentioned objective, by using the field trip and aerial photographs interpretation, 78 landslide points were found and mapping of these landslides were prepared. The data informations about layers of slope, aspect, altitude, slope curvature, lithology, landuse, distance of river, distance of road, distance of fault, index of stream power, wetness index and sediment transport index were considered as effective factors on landslide events; and then theirs maps were digited in GIS field. By using the laws of probability, relations among factors and landslides were considered and mapping of landslide sensitivity areas in region was prepared. Model assessment was carried out by one third of landslide points, SCAI index and ROC curve. The results showed a high correlation between dangerous map and landslide distribution. The probability model precision was estimated as very well $(79.87 \%)$ in study area. The most important innovation of this research, comparing to other researches conducted, is using the factors such as slope curvature, index of stream power, wetness index and sediment transport index, as well as simultaneous employing of two important methods for evaluating the model (SCAI index and ROC curve).
\end{abstract}

Keywords: Landslide, Probability Theory, GIS, SCAI Index, ROC Curve.

* Correspondence Address: College of natural resources and marine science, Tarbiat Modares University (T.M.U), Noor, Mazandaran .Tel: 01226253101 Email: Morady5hr@yahoo.com 